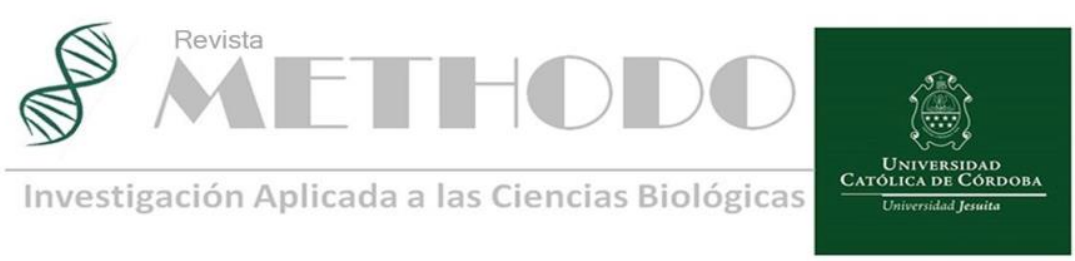

ARTICULO DE REVISION Rev. Methodo 2020;5(2):63-69 https://doi.org/10.22529/me.2020.5(2)05

Recibido 16 Dic. 2019 | Aceptado 27 Ene. 2020 |Publicado 20 Abr. 2020

\title{
Estado actual del uso de medicamentos biológicos en asma grave
}

\section{Biologic therapy for severe asthma: an update}

\author{
Angélica Durán González ${ }^{1}$, Ricardo J. Saranz ${ }^{2}$, Natalia A. Lozano ${ }^{2}$, Graciela Alegre $^{2}$, Paula Robredo², \\ Pilar Visconti ${ }^{2}$, Alejandro Lozano ${ }^{2}$

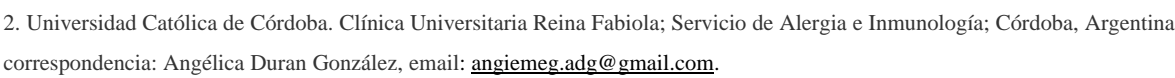

\section{Resumen}

Asma es una enfermedad heterogénea que se manifiesta con diferentes fenotipos y endotipos de fisiopatología compleja. El asma grave resistente al tratamiento con altas dosis de esteroides inhalatorios es una entidad infrecuente pero que plantea un gran desafío para el médico. Los progresos en los conocimientos de la fisiopatogenia del asma han permitido el desarrollo de nuevas terapias con inmunobiológicos que permiten una terapia más individualizada en el contexto de la medicina de precisión. La mayoría de estas nuevas terapias han sido desarrolladas para el asma T2 alta, principalmente eosinofílica, pero son menos los desarrollos para el asma T2 baja, cuyos mecanismos no están completamente dilucidados. Esta revisión intenta recopilar las más recientes evidencias acerca del uso de medicamentos biológicos en el asma grave, con especial énfasis en sus mecanismos de acción, indicaciones, eficacia clínica y posibles efectos adversos.

Palabras clave: Biológicos, anticuerpos monoclonales, asma eosinofílica, omalizumab, polizumab, dupilumab.

\begin{abstract}
Asthma is a heterogeneous disease with a complex pathophysiology that manifests with different phenotypes and endotypes. Severe asthma, resistant to treatment with high doses of inhaled steroids, is an uncommon entity but it implies a great medical challenge. Progress in the knowledge of the pathophysiology of asthma has allowed the development of new and more individualized therapy with biologics in the context of precision medicine. Most of these targeted biologic therapies for have been developed for asthma type-2 high, mainly eosinophilic, but there are fewer developments for non-T2 asthma, whose mechanisms are not completely elucidated. This review attempts to compile the most recent evidence about the use of biologic therapies in severe asthma, with special emphasis on its mechanisms of action, indications, clinical efficacy and possible adverse effects.
\end{abstract}

Keywords: Biologics, monoclonal antibodies, eosinophilic asthma; omalizumab, mepolizomab, dupilumab.

\section{Introducción}

Revista Methodo: Investigación Aplicada a las Ciencias Biológicas. Universidad Católica de Córdoba Jacinto Ríos 571 Bo Gral. Paz. X5004FXS. Córdoba. Argentina. Tel.: (54) 3514517299 / Correo: methodo@ucc.edu.ar / Web: methodo.ucc.edu.ar | ARTICULO DE REVISION Rev. Methodo 2020;5(2):63-69 
El asma es una enfermedad heterogénea caracterizada por una inflamación crónica de la vía aérea, hiperreactividad bronquial y una obstrucción bronquial variable que provoca síntomas característicos tales como disnea, tos y sibilancias.

Asma grave es aquella que permanece mal controlada a pesar del uso de las más altas dosis de medicamentos disponibles, como corticoesteroides inhalables (CEI) a altas dosis más beta2 agonistas de acción larga (LABA) e incluso esteroides orales, o que solo puede controlarse con el máximo nivel de terapia para el asma con un riesgo inaceptable de existencia de efectos adversos ${ }^{1,2,3}$. Se corresponde con los escalones 4 y 5 de Iniciativa Global para el Asma (GINA) ${ }^{1}$. Previo a categorizar al paciente con asma como grave se debe realizar un adecuado diagnóstico diferencial y de condiciones comórbidas, verificar la adherencia al tratamiento y el uso correcto de dispositivos inhalatorios y el control de factores medioambientales y psicosociales. El asma grave ocurre aproximadamente en un 3 a $10 \%$ de los pacientes con asma ${ }^{1,3}$.

En los últimos años, con la introducción de nuevos medicamentos biológicos, se ha avanzado notablemente en el manejo del asma grave, principalmente en su endotipo $\mathrm{T} 2 \mathrm{alto}^{4}$. Son anticuerpos monoclonales específicos que actúan directamente sobre la inmunopatogenia de la enfermedad ${ }^{4}$.El primer biológico aprobado para su uso clínico fue el omalizumab, un anti inmunoglobulina E (IgE). En la actualidad, existen tres medicamentos biológicos aprobados que actúan bloqueando la acción de la IL5 (mepolizumab y reslizumab), o su receptor (benralizumab) y el dupilumab como antirreceptor de IL4 ${ }^{4}$. Los objetivos de la presente revisión fueron recopilar las más recientes evidencias acerca del uso de medicamentos biológicos en el asma grave, su mecanismo de acción, sus indicaciones y eficacia clínica y los posibles efectos adversos.

\section{Materiales y métodos}

Se realizó una búsqueda bibliográfica, en idioma inglés, en base de datos electrónica Medline, considerando como términos de búsqueda "severe asthma", "eosinophilic asthma", "monoclonal antibodies", "biological drugs", "biologicals", "omalizumab", "benralizumab", “dupilumab", "mepolizumab", "reslizumab" de los últimos tres años (2017-2019). Bibliografía previa a dicho intervalo de tiempo sirvió de utilidad complementaria. Se realizó una revisión crítica de cada uno de los artículos que figuran en las referencias.

\section{Fenotipos y endotipos de asma grave}

Actualmente el ama grave no es considerada una enfermedad única, sino un abanico de diversas entidades clínicas y mecanismos fisiopatológicos que se corresponden con distintos fenotipos y endotipos $^{5}$. El fenotipo hace referencia a las características clínicas observables de la enfermedad, mientras que el endotipo se relaciona con el mecanismo biológico subyacente, con biomarcadores específicos. El tratamiento dirigido por biomarcadores es parte de la medicina de precisión cuyo objetivo final es la adaptación de estrategias preventivas y terapéuticas a las características de cada paciente a fin de obtener el mejor resultado clínico individual ${ }^{5,6}$.

Se reconocen dos endotipos de asma: T2 elevado (asma esosinofílica) y $\mathrm{T} 2$ disminuido o No-T2 (asma no eosinofílica $)^{5,7,8}$. Esto explica el hecho de que, a pesar de presentar síntomas similares, los pacientes con asma pueden responder de modo diferente ante las mismas intervenciones terapéuticas. Los pacientes con endotipo T2 tienen eosinófilos elevados en el esputo y las vías aéreas mientras que los individuos con asma T2 disminuido expresan neutrófilos elevados o una inflamación paucigranulocitica, con escasas células inflamatorias, en el esputo y las vías aéreas ${ }^{7}$.

En el endotipo T2 elevado, hay defectos en la barrea epitelial de las vías aéreas, con una marcada disminución de la expresión de E-cadherina y claudina 18 y la consecuente alteración de las uniones estrechas. Esto facilita el ingreso de alérgenos, virus y bacterias a las vías aéreas. Las células epiteliales de las vías aéreas reconocen antígenos mediante los receptores de reconocimiento de patrones (PRR) y secretan diferentes citoquinas como linfopoyetina del estroma tímico (TSLP), IL-25 e IL-33 responsables del inicio de la respuesta inflamatoria T2. ${ }^{5,8}$. La IL-25 e IL-33 activan las células linfoides innatas tipo 2 (ILC2), que son productoras potentes de IL-5 e IL-13, además de activar a las células dendríticas. La IL5 tiene un rol fundamental en la maduración, diferenciación y migración de eosinófilos desde la médula ósea. Las IL-4 e IL-13 promueven la sobreexpresión de células caliciformes, aumentan la producción mucosa y la hiperreactividad bronquial. La IL-4 es producida por las células dendríticas e induce la diferenciación de Th0 en Th2 que, a su vez, secretan IL-4, IL-5 e IL-13. Además, los Th2 activan a los LB, los cuales se diferencian en plasmocitos secretores de Inmunoglobulina $\mathrm{E}$

Revista Methodo: Investigación Aplicada a las Ciencias Biológicas. Universidad Católica de Córdoba. Jacinto Ríos 571 Bo Gral. Paz. X5004FXS. Córdoba. Argentina. Tel.: (54) 3514517299 / Correo: methodo@ucc.edu.ar / Web: methodo.ucc.edu.ar | ARTICULO DE REVISION Rev. Methodo 2020;5(2):63-69 
(IgE) específica de alérgeno, clave en la respuesta celular T2. Los eosinófilos son las principales células de la respuesta T2, y luego de haber sido activados secretan múltiples mediadores inflamatorios como IL-13, IL-5, quemoquinas como eotaxina, cystenil leucotrienos (CysLTS) como LTC4, LTD4, LTE4, proteínas citotóxicas como la proteína básica mayor (MBP), peroxidasa eosinofílica (EPX), proteína eosinofílica catiónica (ECP) y neurotoxina derivada del eosinófilo (EDN). Los eosinófilos promueven la remodelación (en particular, el engrosamiento de la membrana basal) a partir de la activación de los fibroblastos bronquiales, y la broncoconstricción a través de los CysLTS ${ }^{5,8}$. Tanto los mastocitos como los basófilos expresan en su membrana el receptor de alta afinidad para $\operatorname{IgE}(\mathrm{Fc} \varepsilon \mathrm{R} 1)$ y son activados por el entrecruzamiento de 2 de dichos receptores mediante la unión de una mólecula de IgE específica a los mismos. Una vez activados, los mastocitos secretan histamina, PGD2, CysLTs y triptasa propagando la respuesta inflamatoria ${ }^{6}$. El principal biomarcador del endotipo T2 es un número elevado de eosinófilos en las vías aéreas. Un recuento de eosinófilos en esputo mayor a 2$3 \%$ se corresponde con asma alérgica ${ }^{7}$. El recuento de eosinófilos en sangre es un biomarcador menos exacto, aunque más accesible. Un número de eosinófilos mayor a 300-400 cel/microlitro se utiliza como predictor de respuesta a los antagonistas de IL5. En el asma eosinofílica existen niveles elevados de periostina, producida por las células epiteliales y fibroblastos en respuesta a la IL-13 y es característico un aumento en la fracción exhalada de óxido nítrico (FENO). Por último, se utiliza el nivel elevado de $\mathrm{IgE}$ específica para alérgenos como marcador de atopia. Los fenotipos T2 aumentado incluyen: asma alérgica de inicio temprano, asma eosinofílica de inicio tardío en la edad adulta, y la enfermedad respiratoria exacerbada por aspirina (AERD). Algunos autores incluyen otros dos fenotipos: asma inducida por ejercicio y la aspergilosis broncopulmonar alérgica ${ }^{5,6}$.

Los fenotipos correspondientes a la respuesta T2 disminuida son el asma relacionada con obesidad de inicio tardío, el asma paucigranulocítica y el asma neutrofílica. Los endotipos subyacentes al mecanismo $\mathrm{T} 2$ disminuido son poco conocidos $(5,7,8)$. Típicamente la enfermedad inicia en la edad adulta y responde menos al tratamiento con esteroides inhalados. En el asma neutrofílica hay un aumento del recuento de neutrófilos en el esputo probablemente como consecuencia de la activación de la inmunidad innata por virus, bacterias o irritantes medioambientales, a través de células epiteliales, macrófagos, células dendríticas y del factor de transcripción nuclear (NFKB) que inicia la respuesta inflamatoria con producción de IL-8, un potente atrayente de neutrófilos. Las citoquinas implicadas incluyen las producidas por Th1 y Th17: IL-1, interferón gamma (INF gamma) e IL-17. Esta última contribuye a la remodelación de la vía aérea estimulando fibroblastos, y puede promover la producción de IL-8.

El asma paucicelular representa el $40 \%$ del asma no eosinofílica y responde poco al tratamiento con corticoides, probablemente porque presenta escasa inflamación. El asma secundaria a la obesidad constituye un fenotipo de inicio tardío predominante en mujeres, y está asociada a una inflamación predominante neutrofílica o paucicelular. Estos pacientes presentan inflamación relacionada con el tejido adiposo, activación de macrófagos, incremento de IL-6, TNF alfa y leptinas, que puede ser reversible con la pérdida de peso $^{5,8}$.

\section{Medicamentos biológicos}

La mayoría de los medicamentos biológicos actualmente disponibles tienen como objetivos terapéuticos diferentes mediadores inflamatorios de la repuesta T2 alta previamente descriptos ${ }^{7}$. Esta revisión hará foco en el omalizumab (anti $\mathrm{IgE}$ ), mepolizumab y reslizumab (anti IL-5), benralizumab (anti receptor de IL-5) y dupilumab (Anti receptor de IL-4). Por lo tanto, la determinación del endo-fenotipo y sus biomarcadores constituye un paso fundamental para la elección de los medicamentos biológicos en el asma grave. Los mecanismos fisiopatogénicos y sus antagonistas se muestran en la (Figura 1).

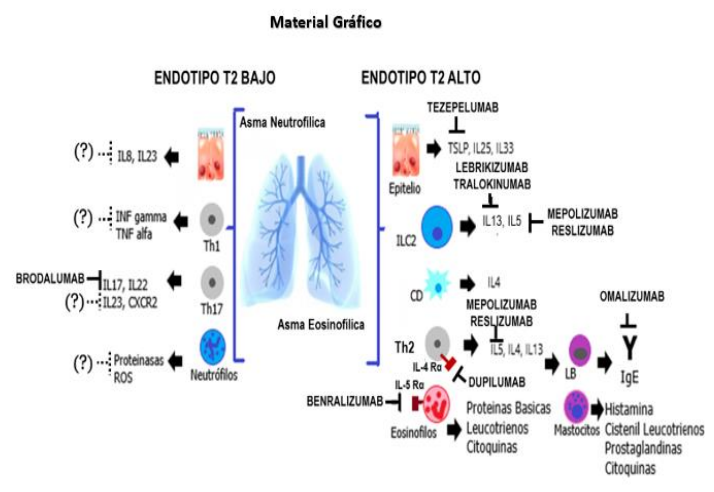

Figura 1. Mecanismos de la inflamación T2 alta y T2 bajo (No T2) en asma y blancos terapéuticos de los inmunobiológicos. (ILC2: célula linfoide innata tipo 2, CD: célula dendrítica; TSLP: Linfopoyetina estromática tímica; IL: Interleucina; CXCR2: Receptor de IL-8; ROS: especies reactivas de oxígeno). (Modificado de Tabatabaian F et al. Immunol Allergy Clin North Am 2017; 37: 329-343).

Revista Methodo: Investigación Aplicada a las Ciencias Biológicas. Universidad Católica de Córdoba Jacinto Ríos 571 Bo Gral. Paz. X5004FXS. Córdoba. Argentina. Tel.: (54) 3514517299 / Correo: methodo@ucc.edu.ar / Web: methodo.ucc.edu.ar | ARTICULO DE REVISION Rev. Methodo 2020;5(2):63-69 


\section{Omalizumab}

El omalizumab es un anticuerpo recombinante humanizado, principalmente $\operatorname{IgG} 1$, que se une a la $\mathrm{IgE}$

sérica, formando complejos inmunes que impiden su unión a los receptores de membrana para IgE de mastocitos y basófilos inhibiendo la cascada inflamatoria pro- alérgica. Además, produce una regulación negativa en la expresión de los receptores para $\operatorname{IgE}$ de alta afinidad ${ }^{9}$.

Fue aprobado por la Food and Drug Administration (FDA) en 2003 para su uso en adultos y adolescentes mayores de 12 años con asma alérgica y en 2016 para niños mayores de 6 años. La European Medicines Agency (EMA) lo aprobó a en 2005 y 2009 para las mismas edades. La Administración Nacional de Medicamentos, Alimentos y Tecnología Médica (ANMAT) lo aprobó en 2009 para el tratamiento del asma alérgica persistente moderada o grave en adultos y niños mayores de 6 años. Por lo tanto, su uso se ha extendido por más de 10 años y es el único biológico aprobado para niños mayores de 6 años ${ }^{7,9}$

Las indicaciones clínicas abarcan asma grave con biomarcadores de alergia tales como $\operatorname{IgE}$ específica positiva para al menos un aeroalergeno e IgE sérica total de 30 a $700 \mathrm{UI} / \mathrm{mL}$ en pacientes mayores de 12 años, y de 30 a $1300 \mathrm{UI} / \mathrm{mL}$ para pacientes de 6 a 11 años (en la Unión Europea está aprobado para un nivel de $\operatorname{IgE}$ de hasta 1500 $\mathrm{IU} / \mathrm{mL})^{9}$.

El omalizumab ha demostrado lograr una reducción en el número y gravedad de las exacerbaciones de asma, disminuye el número de ingresos hospitalarios, mejora levemente la función pulmonar y disminuye el uso de CEI, y en algunos casos, permite suspender los esteroides orales $^{4,9}$. Todos estos efectos se traducen en un mejor control de los síntomas y mejoría de la calidad de vida. En estudios recientes se comprobó un descenso en las exacerbaciones estacionales desencadenadas por infecciones virales mediante la mejora de la inmunidad antiviral, específicamente la producción de interferón tipo $\mathrm{I}^{9}$.

Para el inicio del tratamiento con este biológico es necesario una determinación de la IgE total y de la sensibilización para aeroalergenos mediante prueba de punctura o IgE alérgeno-específica en suero. El fenotipo que responde mejor al omalizumab es el asma con múltiples comorbilidades alérgicas (sensibilización a múltiples alérgenos, dermatitis atópica y alergia alimentaria) asociada a recuentos de eosinófilos séricos mayor a 300 células/microlitro, niveles elevados de IgE total y de $\mathrm{FeNO}^{4}$. La dosis de omalizumab es de $0.016 \mathrm{mg} / \mathrm{kg}$ por UI de $\mathrm{IgE}$ administrada cada 4 semanas. Generalmente se necesitan 150 a $375 \mathrm{mg}$ cada 2 a 4 semanas por vía subcutánea $^{4,9,10}$. El monitoreo terapéutico clínico y no existe ningún biomarcador complementario predictivo de respuesta. Generalmente la respuesta terapéutica se observa luego de 3 a 6 meses de uso.

El omalizumab en general es bien tolerado. Sus efectos adversos más frecuentes son locales y de resolución rápida, como dolor local en el sitio de la inyección y reacciones cutáneas ${ }^{4,9,10}$. Aproximadamente $0.1 \%$ a $0.2 \%$ de los pacientes tratados presentan anafilaxia, la cual se produce generalmente durante las primeras 3 dosis, efecto no observado en niños. Por este motivo la FDA colocó una nota de advertencia en el medicamento y se aconseja otorgar a los pacientes un autoinyector de adrenalina y la educación en el reconocimiento y tratamiento de la anafilaxia9.

\section{Mepolizumab}

El mepolizumab es un anticuerpo monoclonal IgG1 que se une a la IL-5 e impide su enlace a la cadena alfa del receptor para IL-5 en la membrana de eosinófilos y basófilos ${ }^{4}$. Por lo tanto, disminuye el reclutamiento, maduración, diferenciación y activación de los eosinófilos en las vías aéreas y disminuye la expresión del receptor para IL-5 en la membrana de eosinófilos y basófilos ${ }^{7}$.

Fue aprobado por la FDA y EMA en 2015 en una dosis de $100 \mathrm{mg}$ por vía subcutánea, una vez al mes, para el tratamiento del asma eosinofílica grave, en adolescentes mayores a 12 años y adultos. Se utiliza un número de corte de eosinófilos séricos para su inicio de 300 células/microlitro. Si el paciente está bajo tratamiento con corticoides orales crónicos, el número de corte de eosinófilos para el inicio del tratamiento desciende a 150 células/microlitro ${ }^{11}$. El mepolizumab ha mostrado ser eficaz en la reducción del número de exacerbaciones de asma, mejorar el control del asma, la calidad de vida, y la función pulmonar en pacientes con asma eosinofílica grave ${ }^{11}$. Una revisión de Cochrane de 2017 demostró que los pacientes tratados con mepolizumab tenían aproximadamente la mitad de exacerbaciones con requerimiento de esteroides orales en comparación con los que recibieron placebo $^{12}$. El estudio SIRIUS (Steroid Reduction with Mepolizimab Study) ${ }^{13}$ evalúo pacientes con eosinofilia sérica crónica a pesar del tratamiento diario con esteroides orales y mostró que en los pacientes que recibieron mepolizumab se descendió la dosis de corticoesteroides orales 
(OCS) en un $50 \%$ y el $14 \%$ de los mismos lograron discontinuar el tratamiento corticoideo. La disminución en el número de exacerbaciones es proporcional a los niveles de eosinofilia sérica. Los pacientes que no responden adecuadamente a omalizumab cuando rotan al tratamiento con mepolizumab pueden mejorar significativamente el control de los síntomas, su calidad de vida, disminuyen el número de exacerbaciones y el uso de OCS y mejoran el volumen espiratorio forzado en el primer segundo (VEF1) ${ }^{4}$.

Luego de su inicio no es necesario monitorizar el tratamiento con paraclínica sanguínea. Se recomienda un seguimiento clínico a los 3 a 6 meses del inicio del tratamiento para definir su continuidad. En caso de una respuesta positiva el tratamiento se continúa de modo crónico dado que en caso contrario los niveles de eosinofilia sérica comienzan a elevarse luego de 12 semanas de su interrupción $^{11}$.

Generalmente es bien tolerado, los efectos adversos más frecuentes son leves, como cefalea, nasofaringitis y reacciones locales en el sitio de inyección. Se han descripto excepcionalmente reacciones de hipersensibilidad sistémicas agudas y retardadas (ej. anafilaxia, urticaria, angioedema, broncoespasmo e hipotensión) tras su administración. También se ha reportado activación del virus herpes zoster, por lo que en mayores de 50 años se recomienda su vacunación previa $^{4,10,11}$.

\section{Reslizumab}

El reslizumab es un anticuerpo monoclonal IgG4 que se une a la IL-5 sérica impidiendo así la unión a su receptor en la membrana de eosinófilos y basófilos ${ }^{7}$. En 2016 fue aprobado por la FDA y EMA para su uso en pacientes mayores de 18 años con asma grave eosinofílica ${ }^{4}$. Se utiliza un punto de corte de eosinofilia sérica para su uso de 400 cel/microlitro.

Reslizumab ha demostrado disminuir la frecuencia de exacerbaciones, mejorar la función pulmonar con incremento del VEF1, mejorar la calidad de vida, el control del asma y disminuir el recuento de eosinófilos séricos en varios estudios randomizados. Este medicamento reduce las exacerbaciones de asma en un 50 a $59 \%$ comparado con placebo ${ }^{14}$.

Se administra en dosis de $3 \mathrm{mg} / \mathrm{kg}$ durante 20 a 50 minutos, por vía intravenosa, cada 4 semanas. Al igual que para mepolizumab, se recomienda su uso crónico dado que los eosinófilos séricos comienzan a ascender luego de 12 semanas de su discontinuación ${ }^{14}$. Generalmente es bien tolerado, los efectos adversos más frecuentes son leves e incluyen infecciones del tracto respiratorio superior como nasofaringitis y sinusitis. Se han visto casos de anafilaxia secundaria a su uso, por lo que la FDA sugiere, como advertencia, que los pacientes deben ser educados para el reconocimiento y tratamiento de este posible efecto adverso, incluso con el uso de autoinyectores de adrenalina $a^{4,10,14}$.

\section{Benralizumab}

Benralizumab es el último antagonista de IL-5 aprobado por la FDA para el tratamiento del asma grave. A diferencia de los biológicos previamente descriptos, benralizumab ejerce su acción uniéndose directamente a la subunidad alfa del receptor de IL-5 en la membrana de eosinófilos y basófilos e induce apoptosis de dichas células por un mecanismo de citotoxicidad dependiente de anticuerpos ${ }^{4}$. Fue aprobado para su uso en pacientes mayores de 12 años con asma eosinofílica grave a finales de 2017 por la FDA e inicios de 2018 por la EMA. Su uso se restringe a los individuos con un recuento de eosinófilos séricos mayor ó igual a 300 células/ microlitro ${ }^{7}$. Benralizumab ha demostrado ser eficaz disminuyendo la frecuencia de exacerbaciones de asma, mejorando los puntajes de síntomas, mejora la función pulmonar (mejora el VEF1 prebroncodilatador) y disminuye la necesidad de esteroides orales ${ }^{15}$.

Los predictores de respuesta clínica a benralizumab incluyen el uso de corticoides orales, historia clínica de poliposis nasal, función pulmonar disminuida (Capacidad Vital Forzada menor a 65\%), 3 o más exacerbaciones en el año anterior y un recuento de eosinófilos séricos $>300$ cel/microlitro ${ }^{15}$.

Este medicamento se administra en una dosis de 30 mg, subcutáneo, cada 4 semanas durante las primeras 3 dosis y luego cada 8 semanas. La respuesta al tratamiento puede ser evaluada luego de su uso durante 3 a 6 meses. Generalmente es bien, aunque se han observado casos aislados de hipersensibilidad a benralizumab con anafilaxia, angioedema o urticaria ${ }^{4,16}$.

\section{Dupilumab}

El dupilumab es un anticuerpo monoclonal que se une a la subunidad alfa del receptor de IL-4, impidiendo la unión de sus ligandos, la IL-4 y la IL-13 con la consecuente inhibición de la respuesta proinflamatoria mediada por $\mathrm{T}^{7}$.

Este inmunobiológico fue aprobado en 2018 por la FDA para su uso en pacientes mayores de 12 años con asma eosinofílica grave esteroide dependiente ${ }^{4}$.

Revista Methodo: Investigación Aplicada a las Ciencias Biológicas. Universidad Católica de Córdoba. Jacinto Ríos 571 Bo Gral. Paz. X5004FXS. Córdoba. Argentina. Tel.: (54) 3514517299 / Correo: methodo@ucc.edu.ar / Web: methodo.ucc.edu.ar | ARTICULO DE REVISION Rev. Methodo 2020;5(2):63-69 
La eficacia de dupilumab ha sido comprobada en ensayos clínicos controlados, mostrando una disminución en las exacerbaciones de asma (que es mayor en sujetos con un recuento de eosinófilos mayor o igual a 300 células/microlitro y con FENO mayor o igual a $25 \mathrm{ppb}$ ), una mejoría en la función pulmonar con incremento del VEF1, disminución en la necesidad de esteroides orales en un $70 \%$ (incluso con posibilidad de discontinuarlos) y una mejora en los puntajes clínicos del control de síntomas ${ }^{17}$.

Debe ser administrado en dosis de 400 a $600 \mathrm{mg}$ por vía subcutánea, seguido de 200 o 300 mg cada 2 semanas. Las dosis mayores se utilizan en pacientes con dependencia de esteroides orales, o que presentan dermatitis atópica grave concomitante. También ha demostrado ser eficaz en pacientes con rinitis alérgica, sinusitis crónica y poliposis nasosinusal, por lo que es de elección en los pacientes con asma grave eosinofílico que presentan alguna de estas comorbilidades ${ }^{18}$. Es el único biológico que puede ser autoadministrado por el paciente en su hogar luego de un entrenamiento técnico apropiado.

Los efectos adversos más frecuentes con su uso son reacciones en el sitio de la administración (en $18 \%$ de los pacientes) e hipereosinofilia (en 4 a $14 \%$ de los pacientes). La hipereosinofilia es transitoria y asintomática en la mayoría de los pacientes, aunque algunos presentan síntomas de neumonitis y miositis ${ }^{17,18}$.

\section{Biológicos en desarrollo}

Existe una investigación continua de nuevos medicamentos biológicos para el asma grave ${ }^{19}$. Lebrikizumab y tralokinumab son anticuerpos monoclonales que inhiben IL-13 que se encuentran en fases avanzadas de investigación. Tezepelumab es un anticuerpo monoclonal que inactiva la linfopoyetina estromática tímica (TSLP) y se encuentra en la fase 3 de investigación con una eficacia prometedora independientemente de la presencia de inflamación T2. Brodalumab es un inmunobiológico que se une al receptor IL-17RA bloqueando la acción biológica de IL-17A, IL-17F, IL-17A/F e IL-17E (IL-25), se encuentra en estudio de fase 2 con eficacia prometedora por reducción de los síntomas de asma y mejoría de la función pulmonar, y al igual que tezepelumab, podría ser eficaz en pacientes sin evidencia de inflamación T2 para los cuales no existen tratamientos inmunodirigidos aprobados actualmente. Fevipiprant es un inhibidor de CRTH2 (receptor DP2) que se encuentra en la fase 3 de investigación y tiene una eficacia prometedora en pacientes con asma grave no controlada. El inhibidor del receptor tirosina kinasa protooncogen KIT, imatinib, tiene buenos resultados en estudios pre-comercialización. En modelos animales, se está estudiando la inhibición de la enzima específica de ADN GATA 3, expresada únicamente de modo intracelular. Otros objetivos terapéuticos en investigación que aún no tienen resultados publicados son anti IL-33, ant ST2, IL-25 e IL-6. $^{19}$ La información sobre los medicamentos biológicos aprobados y de inminente aprobación se resume en la (Tabla 1).

Tabla 1. Síntesis de los medicamentos biológicos para el tratamiento del asma grave (FDA: Food and Drug Administration; EMA: European Medicines Agency; ANMAT: Administración Nacional de Medicamentos, Alimentos y Tecnología Médica).

\begin{tabular}{|c|c|c|c|c|}
\hline Biológico & $\begin{array}{l}\text { Mecanismo } \\
\text { de acción }\end{array}$ & $\begin{array}{l}\text { Dosis/via de } \\
\text { administración }\end{array}$ & $\begin{array}{l}\text { Biomarcadores } \\
\text { predictivos }\end{array}$ & $\begin{array}{l}\text { Estado } \\
\text { regulatorio }\end{array}$ \\
\hline Omalizumab & $\begin{array}{l}\text { AntilgE } \\
\text { Fec Rl }\end{array}$ & $\begin{array}{l}150 \text { a } 375 \mathrm{mg} \mathrm{cada} \\
15-30 \text { dias por via } \\
\text { subcutanea }\end{array}$ & $\begin{array}{l}\text { lgE elevada en el rango } \\
\text { Sensibilizacionna alérgenos } \\
\text { FeNO elevara }\end{array}$ & $\begin{array}{l}>6 \text { años (FDA, EMAY } \\
\text { ANMAT) }\end{array}$ \\
\hline Mepolizumab & Anti IL-5 & $\begin{array}{l}100 \mathrm{mg} \text { c } 130 \text { dias } \\
\text { por via subcutanea }\end{array}$ & $\begin{array}{l}\text { Eosinofiliosen sangre } \\
\text { Eosinofilosen esputo } \\
\text { FeNO elevada }\end{array}$ & $\begin{aligned}> & 12 \text { a ấos (FDA /ANMAT) } \\
& >6 \text { años (EMA) }\end{aligned}$ \\
\hline Resilizumab & Antill-5 & $\begin{array}{l}3 \mathrm{mg} / \mathrm{kg} \mathrm{g} / 30 \text { dias } \\
\text { por via endovenosa }\end{array}$ & Eosinofilosen sangre & $>18$ años (FDAy EMA) \\
\hline Benralizumab & Anti ll-5 Ra & $\begin{array}{l}30 \mathrm{mg} c \mathrm{c} / 30 \text { dias por } \\
\text { via subcutánea }\end{array}$ & Eosinofilosen sangre & $>12$ años (FDA EMA) \\
\hline Dupilumab & Anti ll-4Ra & $\begin{array}{l}400 \text { a } 600 \mathrm{mg} \\
\text { (inicial) seguido por } \\
200-300 \mathrm{mg} \text { c/15 } \\
\text { dias por via } \\
\text { subcutanea }\end{array}$ & Eosinofilosen sangre & $>12$ años (FDA) \\
\hline Tralokinumab & Antil L- -13 & Subcutánea & Periostina sérica & Fase 2 \\
\hline Librikizumab & Anti IL-13 & Subcutảnea & Periostina sérica & Fase 3 \\
\hline
\end{tabular}

\section{Conclusión}

En los últimos años, con el advenimiento de nuevos medicamentos biológicos, se ha avanzado notablemente en el tratamiento disponible para el asma grave. Todos los medicamentos biológicos aprobados actualmente para el uso en estos pacientes tienen como objetivo terapéutico mecanismos pertenecientes a la inflamación T2 alta. Se trata entonces de una medicina individualizada y dirigida al mecanismo inmunopatológico subyacente de la enfermedad. Todos estos medicamentos han demostrado ser eficaces en la reducción de las exacerbaciones, en el control de los síntomas, mejoría de la calidad de vida de los pacientes y de su función pulmonar. La elección entre los diferentes agentes debiera basarse en la presencia de diferentes biomarcadores como eosinófilos en esputo, nivel de FeNO, eosinofilia periférica e IgE específica, así como las comorbilidades atópicas. Se necesita mayor investigación para determinar con mayor exactitud la duración del tratamiento, su uso en la infancia y en individuos con asma por mecanismos no-T2 para los cuales no existen actualmente medicamentos inmunodirigidos disponibles.

Revista Methodo: Investigación Aplicada a las Ciencias Biológicas. Universidad Católica de Córdoba. Jacinto Ríos 571 Bo Gral. Paz. X5004FXS. Córdoba. Argentina. Tel.: (54) 3514517299 / Correo: methodo@ucc.edu.ar / Web: methodo.ucc.edu.ar | ARTICULO DE REVISION Rev. Methodo 2020;5(2):63-69 


\section{Bibliografía}

1. Boulet LP, Bateman E, Bruselle $G$ et al. Difficult to treat \& severe asthma in adolescents and adult patients. Diagnosis and Management. GINA pocket guide for health professionals. 2019; Abril. 1-38. Disponible en https:/ginasthma.org/wpcontent/uploads/2019/04/GINA-Severeasthma-Pocket-Guide-v2.0-wms-1.pdf [consulta: 26 de septiembre de 2019]

2. Barranco Sanz P, Del Cuvillo Bernal A, Delgado Romero J, et al. GEMA. Guía española para el manejo del asma. 2018; 139160. Disponible

en https://www.gemasma.com/ [Consulta: 27 de septiembre de 2019]

3. Neffen H, Vidaurreta S, Balanzat A, et al. Asma de difícil control en niños y adolescentes estrategias diagnósticoterapéuticas. Medicina (Buenos Aires). 2012; 72: 403-413.

4. Krings JG, McGregor MC, Bacharier LB, Castro M. Biologics for severe asthma: Treatment- specific effects are Important in choosing a specific agent. J Allergy Clin Immunol Pract. 2019; 7(5): 1379-1392.

5. Carr TF, Zeki AA, Kraft M. Eosinophilic and noneosinophilic asthma. Am J Respir Crit Care Med 2018; 197: 22-37.

6. Just J, Deschildre A, Lejeune S and Amat F. New perspectives of childhood asthma treatment with biologics. Ped Allergy Immunol 2019; 2: 159-171.

7. Tabatabaian F, Ledford D.K, Casale TB. Biologics and new therapies in asthma. Immunol Allergy Clin North Am 2017; 37: 329-343.

8. Kuruvilla ME, Lee FE, Lee GB. Understanding asthma phenotypes, endotypes and mechanisms of disease. Clin Rev Allergy Immunol 2019; 56(2): 219-233.

9. Humbert M, Busse W, Hanania NA. Omalizumab in asthma: an update on recent developments. J Allergy Clin Immunol Pract 2014; 2: 525-536.

10. Licari A, Manti S, Castagnoli R et al. Targeted therapy for severe asthma in children and adolescents: current and future perspectives. Paediatr Drugs 2019; 21(4) 215-237.
11. Morjaria JB, Emma R, Fuochi V et al. An evaluation of mepolizumab for de treatment of severe asthma. Exp Opin Biol Ther 2019; 19(6):491-500.

12. Farne HA, Wilson A, Powell C, Bax L, Milan SJ. Anti-IL5 therapies for asthma. Cochrane Database Syst Rev 2017;9:CD010834.

13. Bel EH, Wenzel SE, Thompson PJ, Prazma $\mathrm{CM}$, Keene ON, Yancey SW, et al. Oral glucocorticoid-sparing effect of mepolizumab in eosinophilic asthma. N Engl J Med 2014; 371:1189-97.

14. Li J, Wang F, Lin C et al. The efficacy and safety of reslizumab for inadequately controlled asthma with elevated blood eosinophil counts: a systematic review and meta-analysis. J Asthma 2017; 54: 300-307.

15. FitzGerald JM, Bleecker ER, Menzies-Gow A, Zangrilli JG, Hirsch I, Metcalfe P, et al. Predictors of enhanced response with benralizumab for patients with severe asthma: pooled analysis of the SIROCCO and CALIMA studies. Lancet Respir Med 2018; 6:51-64.

16. Edris A, De Feyter $\mathrm{S}$, Maes $\mathrm{T}$ et al. Monoclonal antibodies in type 2 asthma: a systematic review and network meta-analysis. Respir Res 2019; 20: 1-15.

17. Grey A, Katelaris C.H. Dupilumab in the treatment of asthma. Immunotherapy 2019; 10: 859-872.

18. Sastre J, Dávila I. Dupilumab: A new paradigm for the treatment of allergic diseases. J Investig Allergol Clin Immunol 2018;28: 139-150.

19. Corren J. New targeted therapies for uncontrolled asthma. J Allergy Clin Immunol Pract 2019; 2: 1394-1403.

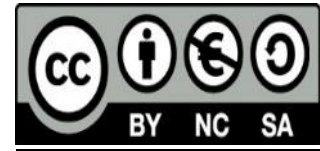

